\title{
Dental Resorptions and their Challenge: Three Case Reports
}

\author{
Luciano Barreto Silva*, Alexandrino Pereira dos Santos Neto, José Alcides Almeida de Arruda, Liana Lins \\ Leite, Lorena do Nascimento Paes Barreto, Pâmella Recco Álvares and Gerhilde Callou Sampaio \\ Universidade de Pernambuco, Brazil \\ Submission: June 13, 2016; Published: July 11, 2016 \\ *Corresponding author: Luciano Barreto Silva, Universidade de Pernambuco, Av. Gen. Newton Cavalcante, 1650 - Aldeia dos Camarás, \\ Camaragibe - PE, CEP: 54753-020, Brazil, Tel: 5581 982888339; Email: lucianobarreto63@gmail.com
}

\begin{abstract}
Dental resorptions, also called root resorptions, are c! onsidered a challenge for odontology mainly because of the complexity and interactions involved in this process that destroys the roots of the teeth. Although clinically this condition may be relatively easy to identify, biologically there are still many open questions concerning the immunologic mechanisms in search of a way to prevent it by the establishment of an early diagnosis. This article gives an overall view of root resorptions while describes three case reports of internal, external and idiopathic resorptions.
\end{abstract}

Keywords: Allergy and immunology; Tooth resorption; Root resorption

Abbreviations: ABO: Brazilian Association of Odontology; PDL: Periodontal Ligament; APCs: Antigen Presenting Cells

\section{Introduction}

Dental resorptions have been a challenge for odontology since Michael Blum first described it in 1530 in the city of Leipzig in Germany. Nevertheless, due to its complexity and the integration with the immunologic system, its scientific comprehension is considered recent, embracing nearly three decades. Root resorptions are classified, in general, as physiologic and pathologic, being the former related to the natural and expectable resorption of the deciduous teeth, and the latter related to pathologic resorptions involving the roots of permanent teeth. On the other hand, pathologic root resorptions are subdivided in two major groups namely: external and Internal.

External root resorptions of permanent teeth are multifactorial, and are usually associated with well known factors, commonly related to inflammatory process caused by trauma, orthodontic therapy and transplantation and reimplantation procedures. Physical pressure over the dental structures and impacted teeth; dental trauma in its various modalities, benign or malignant tumors as well as systemic conditions, such as hormonal alterations have also been featured as possible causes. Another case of external root resorptions, however, has also been described in the literature as Multiple Idiopathic External Root Resorption. Nevertheless, this sort of root resorption associated with unknown causes is a rare finding in dentistry, and its denomination refers to the fact that the triggering factor is not known. This paper insights three cases of root resorptions.

\section{Case Report}

\section{Internal root resorption}

An 11-year-old male patient looked for dental help in the Brazilian Association of Odontology (ABO-Pernambuco) with a previous clinical history of trauma affecting the maxillary upper teeth (Figure 1). Central incisors are usually the most affected teeth during facial trauma. Their position in the maxilla makes them more vulnerable than other teeth; and is exactly what happened to tooth 12 and 11 in this report. Tooth 12 was restored with composite resin right after the accident, while tooth 11 had a fracture in the mesial side of the crown which exposed the pulp and caused pulp necrosis. The teeth went on untreated until the moment of the dental appointment and they both were tender to vertical percussion and responded negatively to cold sensitivity test (EndoFrost).

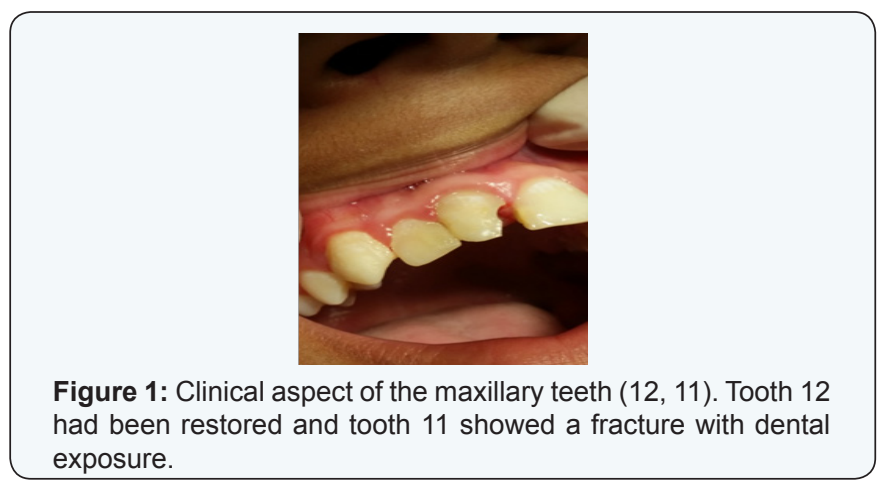


At the radiographic examination, an oval radiolucent image could be observed in the middle third of tooth 12 , associated with a periapical radioluscence (Figure 2). Both teeth had pulp necrosis and periapical lesions and the endodontic treatment was indicated. Due to the oval resorption in the middle third of tooth 12, it was decided to use Schilder technique for obturation, while tooth 11 was obturated with the aid of MacSpadden compactors.

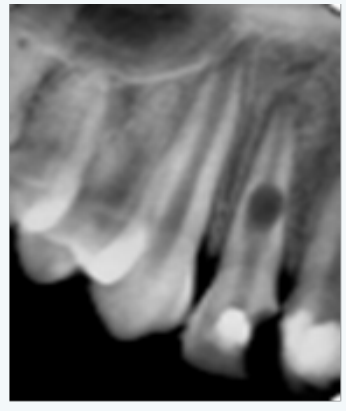

(A)

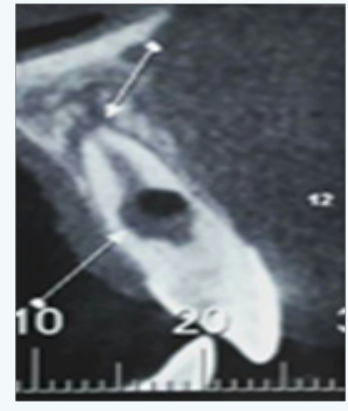

(B)

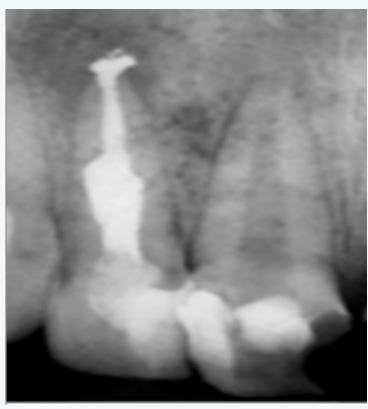

(C)

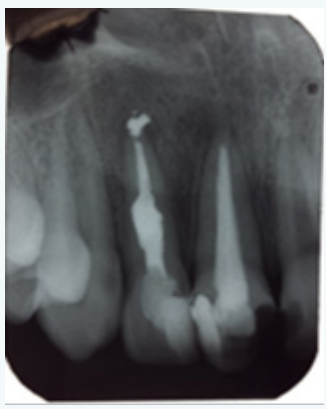

(D)

Figure 2: Radiographic and tomografic images

Figure 2A: Round radioluscent image located in the middle third of tooth 12 campatible with internal root resorption.

Figure 2B: Cone beam cut showing a round to oval image compatible with internal root resorption, and apical image compatible with periapical lesion.

Figure 2C: Endodontic treatment of tooth 12.

Figure 2D: Endodontic treatment of tooth 11.

\section{External root resorption}

A 32-year-old patient looked for endodontic assistance because of a cavity that destroyed half of the crown of the upper maxillary right canine. After the periapical radiograph, a radiolucency could be detected on the apex of the mentioned tooth and laterally in the periodontum, compatible with external root resorption associated with a periapical lesion. Initially, the image in the periodontum was compatible with external root resorption affecting only the external part of the root. The patient was assymptomatic and only searched for dental assistance because of aesthetic matters. The endodontic treatment was then accomplished and revealed a communicating root resorption which seemed to have been originated externally progressing into the root canal. After the obturation using MacSpadden compactors, the area was filled with gutapercha and the patient was instructed for follow up procedures, but never returned to preservation (Figure 3).

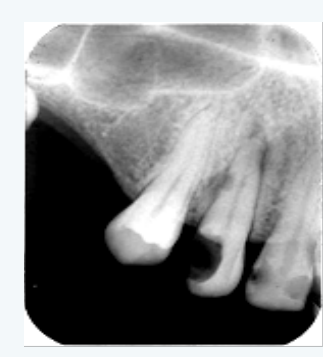

(A)

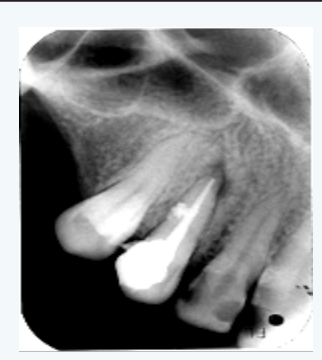

(B)
Figure 3: External root resorption.

Figure 3A: Radiolucency detected on the apex of tooth 13 and laterally in the periodontum, compatible with external root resorption associated with periapical lesion.

Figure 3B: After endodontic treatment. Obturation showed a communicating external root resorption filled with gutapercha.

\section{Idiopathic root resorption}

A 17-year-old healthy Brazilian boy searched for the endodontic department of a private speciality school (ABOBrazilian Association of Odontology) sent by his orthodontist for endodontic evaluation. The patient had never been treated orthodontically, and his main complaint was aesthetical, caused by the dental crowding located in the upper anterior teeth. The clinical intraoral examination reviewed full permanent dentition with one small carious cavity in tooth 16 , and an amalgam restoration on tooth 26 (Figure 4). Dental crowding was also present on the mandibular anterior teeth, as well as crossbite on the lateral upper incisors $(12 ; 22)$. As for what concerned trauma, his report was uneventful. Haematological examinations were within normal aspects as for what concerned hyperparathyroidism, hypoparathyroidism, hyperphosphataemia and hypophosphataemia, and Paget's disease. The patient had a normal growth curve and did not show signs of syndromes such as Papillon-Lefreve, whose main characteristics are premature dental loss and palmar-plantar hyperkeratosis. Altogether, all of his clinical and radiographic examinations led to the diagnosis of multiple idiopathic root resorption.

The patient had no complaint of tooth mobility or any other symptom. All he wanted was to start the orthodontic treatment in order to correct a cross bite involving tooth 12 and 22 caused by dental crowding (Figure 4). However, on the panoramic view, multiple apical root resorption was accidently found, involving the upper and lower teeth (Figure 4). Due to the panoramic radiograph findings, full mouth periapical radiographs were requested for more accurate visualization (Figure 5). (Include RXs). With the diagnosis of multiple apical external root resorption, the orthodontic therapy was contraindicated and the patient is under follow up procedure. 


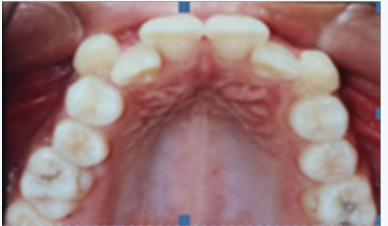

$(4 \mathrm{~A})$

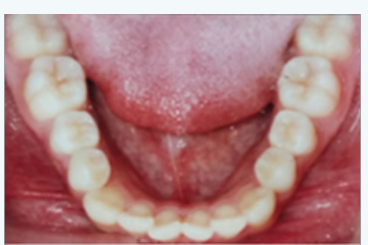

(4B)

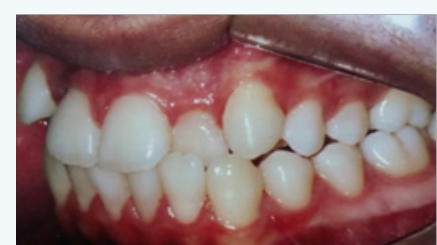

(4C)

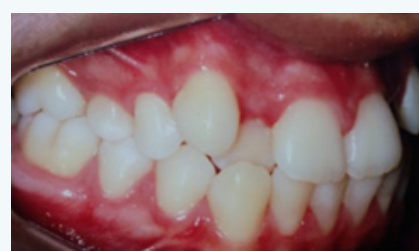

(4D)

Figure 4: Maxillary teeth

Figure 4A: A dental cavity (16) and an amalgam filling (tooth 26) can be observed.

Figure 4B: Mandibular teeth with anterior dental crowding.

Figure 4C, 4D: Cross bite of teeth 12 and 22.

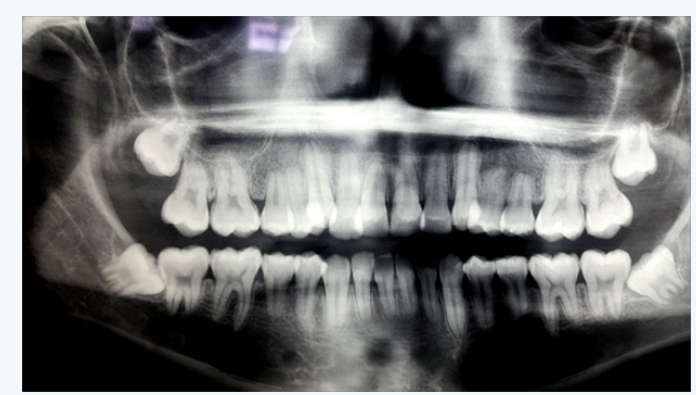

Figure 5: Panoramic view showing apical root resorption involving multiple upper and lower teeth: $11,13,14,15,21,23$, $24,25,31,32,34,35,36,41,42,44,45,46$ and 47.

\section{Discussion}

Up to now, the early detection and the due treatment along with follow up procedures concerning root resorption seems to be the best form of treatment for root resorptions [1]. From the point of view that root resorption is usually asymptomatic, it is advisable to accomplish radiographic and tomographic examinations in patients more prone to develop them, such as patients under orthodontic therapy or who have been victimized by trauma. Although panoramic radiographs can offer a good general view of all teeth and other structures in the face, they are not indicated for definite diagnosis of root resorption under any pretext; due to distortions inherent to this kind of radiograph which may lead do misdiagnosis, not to mention the limitations of details, which can be poorly seen in panoramic views; and therefore periapical radiographs are then indicated.

It is believed that dietary habits may have some influence on the balance of bone turnover, and may influence on the progress of root resorptions. The work of Alam et al. [2] evaluated the fatty composition of arachidonic acid in the alveolar bone of laboratorial rats and concluded that specific dietary lipids altered the fatty acid composition of bone lipids, and added that diets enriched with fish oil decreased the concentration of arachidonic acid by diminishing the amount of osteoclasts in the area investigated. Arachidonic acid can be converted to series 2 prostaglandins or to series 4 leukotriens by ciclooxy-genase pathways. Prostaglandins are known to be hyperalgic and precursor of inflammatory mediators associated with the complement system. Another work [3] studied the influence of $n-3$ polyunsaturated fatty acid on experimental tooth movement. The animals were fed a purified diet containing $10 \%$ refined fish oil while the control animals were fed a diet containing $10 \%$ corn oil. They concluded that the number of osteoclasts on the experimental group nearly $60 \%$ of that seen in controls, and that the degree of bone resorption was $80 \%$, suggesting that enriched fish oil diets may reduce osteoclastic number, diminishing the degree of root resorption and the consequent dental movement. Thus, there seems to be correlation between nutrition and osteoclastic recruitment. Pertinent as it may seem, none of the patients in this study had nutrition problems and had common dietary habits. Although case report 1 showed a patient with pulp necrosis and internal root resorption, its development depends on the blood flow of a living pulp many times, during the process.

\section{Overview}

Root resorption can be described as the destruction of the dental roots by competent cells from the immunologic system. The teeth can be considered as specialized sensory organs which possess some of the hardest tissue in the human body: enamel, located in their crowns, which protects the dentin beneath from the outer environment. The roots of the teeth however, are protected by the periodontum, constituted by alveolar bone, periodontal ligament (PDL) and cementum, while the pulp lies in the middle of the tooth and is responsible for nutrition, dentin formation and sensorial purposes, responding basically with pain to all kinds of stimulus. These three hard tissues show some similarities in their formation. Cementum is an avascular mineralized tissue that covers and protects the root surfaces of the teeth as a whole, occupying the interface between dentin and the periodontal ligament. Not only does the cementum help anchor the tooth to its alveolar bone as it also plays a crucial role to protect the integrity of the root surfaces. Although enamel is not a connective tissue and has no collagen involved in its makeup, its construction follows many of the principles involved in the formation of hard connective tissue $[4,5]$. Once the teeth have been formed as they erupt, they bear the burden of supporting pressures applied over them due to their morphology, function, and their position in the maxillaries. Nevertheless, sometimes physical, chemical or 
mechanical stresses may be strong enough as to cause transitory or permanent damages which may lead to root resorptions.

Internal root resorptions: Internal root resorptions are not as frequently found as the external ones. They seem to be related to pulp aggression, leading to focal necrosis of the odontoblasic layer inside the root canal space without pulp necrosis [6,7]. There seems to be the transformation of normal pulp tissue into granulomatous tissue with the presence of giant multinucleated cells, which are formed through asynchronous fusion of mononuclear cells belonging to the macrophage lineage and originating from the hematopoietic system, which usually appears under the light microscope with 2 to 10 nuclei. Such transformation is believed to be originated from continual bacterial stimulation over a coronal pulp, and worsened when, concomitantly, there are other factors such as dental cavities, dental filling with premature contact or even occlusal trauma [8].

Nevertheless, when observed in periapical radiographs, the image observed is usually a round radiolucent enlargement of the root canal space, typically compatible with internal root resorption and ,for that specific characteristic, easily diagnosed [9]. The stimulus may be strong enough to alter a living pulp to progress into chronic dental pulp inflammation, unleashing the discontinuity of the odontoblastic layer, and therefore exposing dentin antigens which will cause an autoimmune reaction, which frequently starts from the coronal third [10], and may progress to total necrosis, where it's also a common finding the presence of communication with the periodontium in the radiolucent spot [11].

As the process progresses, internal root resorption can be perforating or non-perforating. Differential diagnosis is to be made with external resorptions that communicates with the root canal space. In such situation, the round radioluscence is not that typical and when exists is usually irregular. The clinical pattern is usually asymptomatic, however, it may include the presence of a pink spot, which represents the granulation tissue showing through the resorbed area in the inside. Radiographs are mandatory for diagnosing internal resorption, and more recently the cone beam tomography has been recommended as well, which may reveal a round-to-oval radiolucent enlargement of the pulp space being gradually resorbed $[12,13,1]$. The evolution of internal root resorptions may be jeopardized when there is the presence of caries or fractrures in the tooth affected, opening access to bacterial penetration and consequent pulp necrosis, impeding the circulation and promoting periapical lesions, which implies in more bone destruction and the possibilities of acute or chronic periapical alterations.

External root resorptions: External root resorptions, on the other hand, are much more easily found in routine panoramic or periapical radiographs. Surprisingly as it may seem, permanent teeth survive well throughout the life of a mammalian, under normal conditions, without being destroyed by immune competent cells, even being placed within the alveolar bone full of active cells in an intense metabolism [14]. Nevertheless, triggered by one or more specific factors, external root resorption may appear by a complex interaction of the first lineage of inflammatory cells which may activate more sophisticated ones. Among them, there are very specific antigen presenting cells (APCs) named clasts, which are didactically subdivided in osteoclasts, dentinoclasts and odontoclasts, depending on to the structures that they resorb. The structural, organizational and functional differences between odontoclasts, dentinoclasts and osteoclasts are insignificant, although detectable as for what concerns their size.

Odontoclasts are somewhat smaller. In general, clasts, during active external root resorption, occupy shallow depressions, designated as Howship's lacunae, where their potential acidic production takes place; they are rich in acid phosphatases as well as other lysosomal enzymes; however and surprisingly, they do not function with lysosomal structures as in most other traditional cells. A recognized feature of the clasts is the presence of a proton pump associated with the ruffled border, pumping hydrogen ions into the sealed compartment and thus intensifying the acid environment $[15,16]$. For the fact that clasts are APC cells, they expose the dentinary antigens in their cell membrane and present them to T-lymphocytes which starts immune reactions with the recruitment of B-cells responsible for antibody formation.

Didactically, there have been designation of many types of external root resorptions, being external inflammatory root resorption the most common one, which is usually assymptomatic and arises from trauma, orthodontic movement or by chronic infection of the dental pulp or periodontum [17]. Especifically, as for what concerns orthodontic therapy, more risks are involved since the basis of dental movement depends exclusively on induced bone resorption and new bone formation. Root resorptions are designated as Replacement Root Resorption when the outcome of the stimulus is the substitution of the periodontal ligament (PDL) for osseous tissue, with consequent loss of the tooth.

Idiopathic external root resorptions: Idiopathic external root resorptions is a kind of external root resorption, and is a rare condition that can affect one single tooth, a group of teeth, or multiple ones; usually associated with local or systemic factors. Theoretically, many situations would be able to trigger this rare sort of resorption. Among them, local conditions and the systemic ones. Local conditions can be represented by orthodontic therapy, reimplantation of teeth, occlusal stresses, cysts or tumors, being the most common causes of external resorptions, while the latter would comprise hormonal problems such as hyperparathyroidism, hypoparathyroidism; or syndromes such as Goltz syndrome, Papilon-Lefèvre syndrome or even Turner syndrome [18]. As a matter of fact, there are two most affected regions of the tooth described in the literature: cervical, which begins cervically into the dental pulp direction, and apical, whose resorbing direction progresses apically to the crown of the teeth, usually shortening 
and rounding the roots affected. The term idiopathic is used when the etiological factor that triggered the process can not be identified, along with other clinical characteristics; such as normal clinical appearance of the teeth and the periodontal surrounding tissues, along with normal clinical alveolar bone levels and commonly found as incidental findings on radiographs or tomography.

\section{References}

1. Patel S, Kanagasingam S, Pitt Ford T (2009) External cervical resorption: a review. J Endod 35(5): 616-625.

2. Alam SQ, Kokkinos PP, Alam BS (1993) Fatty acid composition and arachidonic acid concentrations in alveolar bone of rats fed diets with different lipids. Calcif Tissue Int 53(5): 330-332.

3. Iwami-Morimoto Y, Yamaguchi K, Tanne K (1999) Influence of dietary n-3 polyunsaturated fatty acid on experimental tooth movement in rats. Angle Orthod 69(4): 365-371.

4. Bachra BH (1970) Calcification of connective tissue. Int Rev Connect Tissue Res 5: 165-208.

5. Limeback H (1991) Molecular mechanisms in dental hards tissue mineralization. Curr Opin Dent 1(6): 826-835.

6. Consolaro A, Consolaro RB, Prado RF (2005) Reabsorção interna: uma pulpopatia inflamatória. Maringá: Dental Press, pp. 572-594.

7. Nascimento GJF, Emiliano GBG, Silva IHM, Carvalho RA, Galvão HC (2006) Mecanismo, classificação e etiologia das reabsorções dentárias. Rev Fac Odontol Porto Alegre 47(3): 17-22.
8. Çaliskan MK, Türkün M (1997) Prognosis of permanent teeth with internal resorption: a clinical review. Endod Dent Traumatol 13(2): 75-81.

9. Jacobovitz M, de Lima RK (2008) Treatment of inflammatory internal root resorption with mineral trioxide aggregate: a case report. Int Endod J 41(10): 905-912.

10. Silveira CF, Silveira LFM, Martos J (2008) Tratamento endodôntico de incisivos centrais superiores com reabsorção radicular interna - caso clínico. Rev Endod Pesq Ens On Line 4(7): 1-6.

11. Wedenberg C, Lindskog S (1985) Experimental internal resorption in monkey teeth. Endod Dent Traumatol 1(6): 221-227.

12. Tronstad L (1988) Root resorption-etiology, terminology and clinical manifestations. Endod Dent Traumatol 4(6): 241-252.

13. Silveira FF, Nunes E, Soares JA, Ferreira CL, Rotstein I (2009) Double 'pink tooth' associated with extensive internal root resorption after orthodontic treatment: A case report. Dent Traumatol 25(3): 43-47.

14. Ingle, Bakland, Baumgartner: Pathologic tooth resorption. Ingle's Endodontics ( $6^{\text {th }}$ edn), BC Decker inc 2008, pp. 1358.

15. Bawden JW (1989) Calcium transports during mineralization. Anat Rec Malden 224(2): 226-233.

16. Boskey AL (1991) The role of extracellular matrix components in dentine mineralization. Crit Rev Oral Biol Med 2(3): 369-387.

17. Benenati FW (1997) Root resorption: Types and treatment. Gen Dent 45(1): 42-45.

18. Moazami F, Karami B (2007) Multiple idiopathic apical root resorption: a case report. Int Endod J 40(7): 573-578. 